\title{
PYKNODYSOSTOSIS - COMPLICATIONS AFTER DENTAL EXTRACTION
}

J. Bahadun, N.N. Nik-Hussein, N.N.N. Yunus.

Pyknodysostosis - complications after dental extraction. Annal Dent Univ Malaya 2007; 14: 26-30.

\begin{abstract}
Pyknodysostosis is a rare sclerosing bone disorder with autosomal recessive mode of inheritance. The condition is characterized by continuous bone deposition without physiological resorption, occurring mainly in the trabecular and not the cortical bone. The skeletal features include short stature, brachycephaly, open cranial sutures and fontanelles, diffuse osteosclerosis and obtuse mandibular angles. Among the oral features observed were grooved or furrowed palate and disturbances in eruption and exfoliation of teeth. Multiple fractures of long bones and osteomyelitis of the jaw are frequent complications. These are attributed to the increased brittleness and reduced vascularity of the bone as a result of continuous endosteal bone deposition. Treatment is usually successful with surgical management and sustained bactericidal antibiotic therapy, although hyperbaric oxygen has been recommended as adjunct treatment in the more refractory form.
\end{abstract}

Key words: pyknodysostosis; osteomyelitis; complication; extraction; antibiotics

\section{INTRODUCTION}

Genetic skeletal dysplasias represent a heterogenous group of abnormalities of the skeletal system; classified primarily on the basis of the clinical and radiographic findings.

Pyknodysostosis is a rare skeletal dysplasia that derives its name from the Greek word "pyknos" meaning dense. Historically, it was thought to be an atypical presentation of cleidocranial dysostosis and osteopetrosis. Up to 1991 , there were only 130 reported cases worldwide, reflecting the rarity of the condition (1).

The syndrome has an autosomal recessive mode of inheritance and parental consanguinity has been recognized and accepted as a causative factor (2) and the defect has been located to chromosome 1q21 (3).

Until recently, the exact disturbance of the condition was not well understood. However, cellular studies have shed some light into the matter. Karkabi et al (4) reported that patient with pyknodysostosis has normal number of osteoclasts, thus suggesting an impairment of function.
Case Report

J. Bahadun ${ }^{1}$, N.N. Nik-Hussein ${ }^{2}$, N.N.N. Yunus ${ }^{3}$

${ }^{1}$ Dental Consultant Clinic

Sarawak General Hospital, Kuching, Sarawak Malaysia

${ }^{2}$ Department of Children's Dentistry and Orthodontics

Faculty of Dentistry, University of Malaya

50603 Kuala Lumpur, Malaysia

Tel: +603-79674802/63

Fax: $+603-79674530$

E-mail:nnoriah@um.edu.mv

${ }^{3}$ Department of Paediatric Dentistry

Institute of Paediatric, Hospital Kuala Lumpur

Kuala Lumpur, Malaysia

Corresponding author: Prof. Dr. Nik Noriah Nik-Hussein

Furthermore, the osteoclasts appear normal in appearance, their attachment to the bone surface as well as normal ruffled border (5). However, these osteoclasts were found to be deficient in cathepsin$\mathrm{K}$, which is a major bone-matrix resorbing protease. Large vacuoles of collagen fibrils were observed within the cytoplasm, indicative of a defect of the lysosomal degradation of the bone organic matrix.

The characteristic skeletal features of the condition consist of dwarfism (frequently measuring less than $150 \mathrm{~cm}$ ), osteopetrosis, abbreviated terminal phalanges (acro-osteolysis), clavicular dysplasia and hypoplasia of mandibular angles (6). Other features may include narrow shoulders, partial or complete aplasia of the clavicles, exophthalmus and history of fractures in response to minimal trauma. The head is usually large with fronto-parietal bossing, open fontanelles, hypoplastic malar and maxillary bones, beaked nose and obtuse mandibular gonial angles (7).

Intraoral features commonly seen include grooved or furrowed palate, persistence of deciduous teeth, premature or delayed eruption of permanent teeth, malalignment of teeth and enamel hypoplasia (8).

Osteomyelitis of the jaws and recurrent fractures of the long bones are characteristic complications of pyknodysostosis (9). The increased bone deposition increased its brittleness making it highly susceptible to fracture. At the same time, the decrease in vascularity would compromise the patient's local immune response (10). 


\section{Case Report}

A 12 year old boy presented to the clinic complaining of pain and swelling in relation to socket of lower right first molar. The history was unclear as to the management before and immediately after the dental extraction. However, it was noted that the patient had a course of antibiotic prior to extraction but none after the procedure. The tooth was extracted about seven months previously due to abscess and patient has had several recurrent episodes of pain and swelling, which subsided after taking antibiotics.

Medical history revealed that he was diagnosed with pyknodysostosis when he was two years old. He had sustained several fractures of the lower limbs before nine years of age; all in response to minimal trauma. He gave a history of tonsillectomy and adenoidectomy that was done at nine years of age for obstructive sleep apnea. Currently, he is on threemonthly follow-up at the Respiratory Department of Paediatric Institute, Kuala Lumpur Hospital.

The child was the first of three siblings and the youngest sister had also been diagnosed with the same condition. He was born of consanguineous marriage. The patient's mother informed that her brother's twin daughters were also affected by the same condition.

The patient had short stature and was only 129.5 $\mathrm{cm}$ in height and weighed $30 \mathrm{~kg}$. He has frontal bossing, exopthalmus, low-set everted ears and hypoplastic mandible (Fig. 1). His fingers were short and broad (Fig. 2).

Radiographically, there was generalized skeletal sclerosis accompanied by delayed closure of the sutures and fontanelles (Fig. 3). The orthopantomogram showed obtuse mandibular angle and extensive bone loss over the right body of mandible (Fig. 4).

Examination of the complaint site showed bony sequestrum on the lingual side associated with socket of 46 (Fig. 5). The clinical and radiographic findings were consistent with chronic osteomyelitis. The patient was then scheduled for surgical debridement of the wound under general anaesthesia and arrangements were made for him to be admitted for blood investigations and anaesthetic review.

Intravenous (IV) Cloxacillin 500mg and IV Dexamethasone $4 \mathrm{mg}$ were given. A lingual mucoperiosteal flap was raised at 46 region and the infected area was exposed. No discharge was observed from the site but the sequestrum that was loosely attached to the bone was easily removed. The jagged bony edges were then filed and the bony defect curettaged. Copious saline irrigation was flushed into and around the area. Subsequently, two gentamicin-impregnated polymethylmetracrylate (PMMA) beads (hereafter referred as gentamicin beads) were placed in the defect and secured with 4.0 Vicryl sutures. Finally, the flap was repositioned and sutured. A button-hole defect on the lingual side of the area caused by the exposed sequestrum was noted and repaired. Periodontal dressing was placed at the operation site to protect the wound. Ryle's

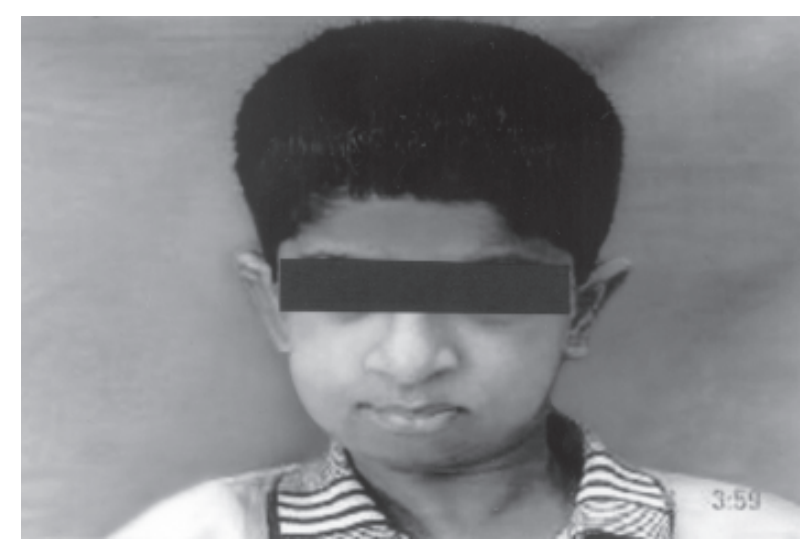

Figure 1: Frontal View of patient.

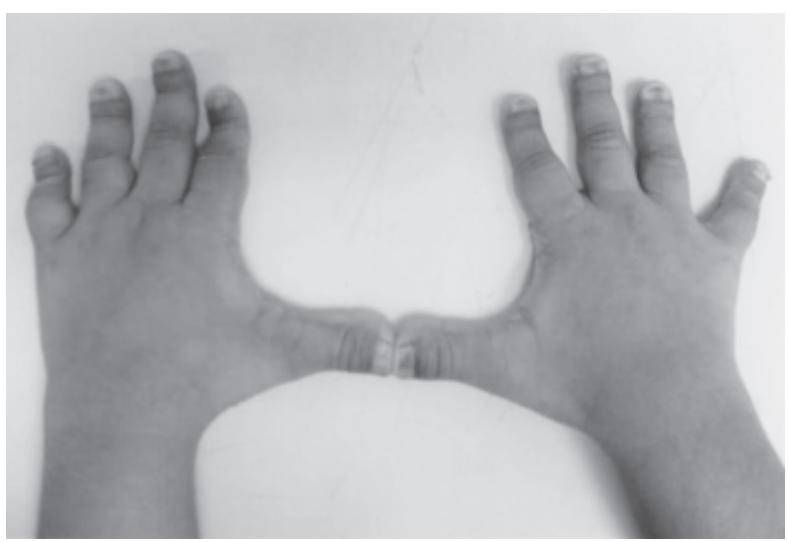

Figure 2: Short and broad fingers.

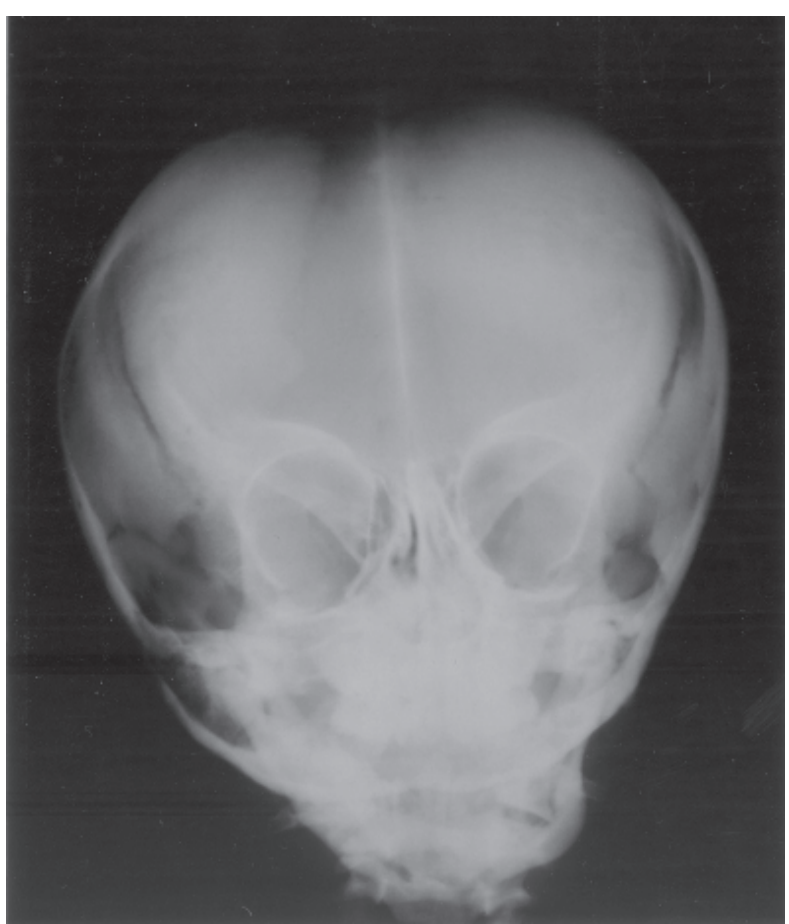

Figure 3: Radiograph showed generalized skeletal sclerosis and delayed closure of the sutures and fontanelles. 


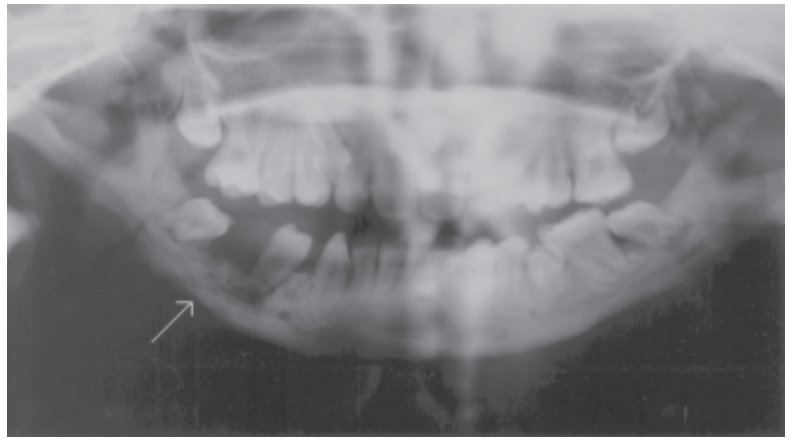

Figure 4: OPG showed obtuse mandibular angle and extensive bone loss over the right body of mandible.

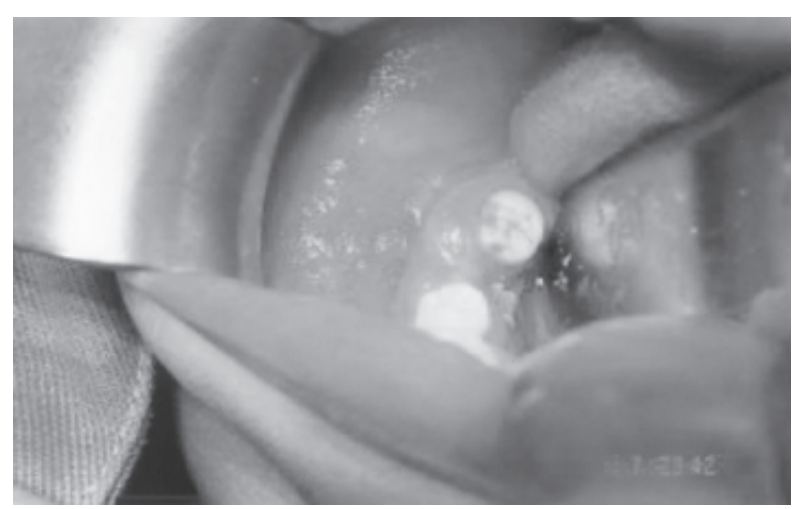

Figure 5: Bony sequestrum on the lingual side associated with socket of 46 .

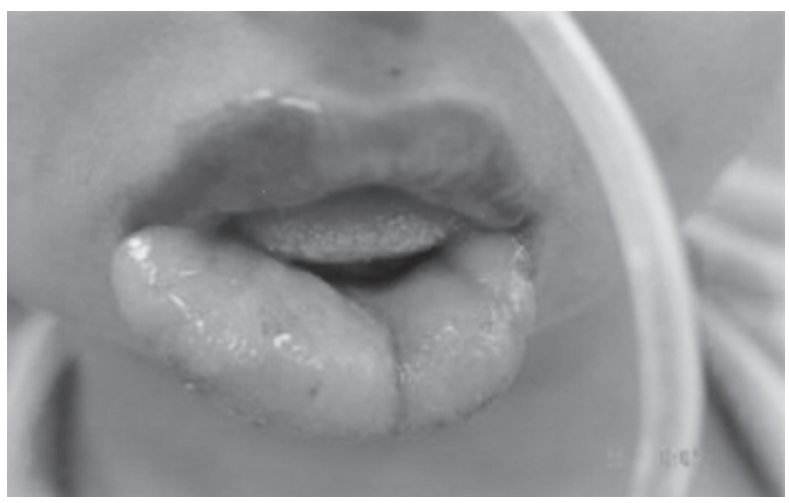

Figure 6: Markedly swollen and everted lower lip.

tube was inserted to avoid contaminating the operation site by oral intake.

The patient was reviewed later in the day and appeared in stable condition. Liquid diet and antibiotic therapy IV Cloxacillin $250 \mathrm{mg} 6$ hourly was prescribed for two weeks. The patient was then reviewed daily. The intraoral area was flushed with Chlorhexidine $0.2 \%$ two times daily and wound healing progressed well.

Subsequently, the Gentamicin beads were then removed under GA seven days after the initial operation. At a review session that evening, it was noted that the patient was very weak and dizzy and was unable to stand. He did not show shortness of breath and no abnormalities was observed on other areas of the body. His lower lip was markedly swollen and were everted (Fig. 6). Intraorally, there was no swelling or redness of the oral mucosa.

The patient was then immediately referred to the paediatrician on-call for further investigation of the condition, possibility of an allergic reaction. He was given IV Chlorpheniramine $4 \mathrm{mg}$ stat, followed by an 8 hourly oral dose of $2 \mathrm{mg}$.

The child's general condition improved dramatically the next day. He was cheerful and was playing with other patients in the ward. The swelling of the lips had reduced markedly. The patient was then referred to the dermatologist for further investigation regarding his allergic reaction.

The child was reviewed daily and IV Cloxacillin $250 \mathrm{mg} 6$ hourly was continued and feeding of liquid diet continued through Ryle's tube. Intraorally, the surgical site was healing well and the child was subsequently discharged at Day 14 . The child and the parents were advised to keep the surgical site clean and they were shown the proper way to clean the area.

He was reviewed one week, two weeks then monthly for three months, after discharged. The surgical site healed well and there were no further episodes of pain and swelling.

The result of the dermatological testing showed that he is not allergic to latex and the test results for anaesthetic agents was inconclusive, thus parents have been advised that further investigations are required to avoid similar incidents in the future.

\section{DISCUSSION}

Pyknodysostosis is a rare disorder and the condition has been reported in patients from the age of nine months up to adults of 77 years of age (11). In this case, the condition was diagnosed when the patient was two years old.

In the past, pyknodysostosis was thought to be a variant of osteopetrosis. This is not surprising as they share some very similar features (2). In both conditions, bone deposition continues without normal physiological resorption. Thus, increased radiodensity, increased osseous fragility as well as frontal bossing are features commonly observed (12).

However, in pyknodysostosis, the increase of bone density occurs mainly in the trabecular and not the cortical bone (4), whereas in osteopetrosis, continuous bone deposition takes place in both trabecular as well as medullary spaces. Hence, the primary differentiating feature between these two conditions is the state of medullary cavities. In pyknodysostosis, the medullary cavity is present with active haemopoiesis. In contrast, in osteopetrosis, reduced medullary space leads to anemia and subsequently causes extramedullary haemopoiesis. In addition, constriction of the cranial foramina results in cranial nerve compression with subsequent alterations in vision and auditory functions (10), which is not seen in pyknodysostosis. 
Generalized skeletal sclerosis is a recognized characteristic of pyknodysostosis, thus, even minor surgical procedure like tooth extraction can be complicated by pathological fracture and osteomyelitis. These events are attributed to the increased brittleness and reduced vascularity of the bone as a result of continuous endosteal bone deposition. In view of these events, perhaps the patient would have benefited from close follow-up and antibiotic coverage post-operatively.

Treatment of chronic osteomyelitis of the mandible is difficult but should include drainage, sequestrectomy and antibiotic therapy (13). Removal of affected bone has three main purposes: to reduce necrotic and avascular bone, to reduce bacterial counts and to promote revascularization by bringing well-vascularised soft tissue into contact with the exposed surface of the diseased bone.

In cases of osteomyelitis of the jaws secondary to sclerosing bone disease, the osteomyelitis is often refractory (14). For this reason, some authors recommended hyperbaric oxygen as an adjunct to surgery and antibiotics (15).

The pathogenesis of osteomyelitis of the jaws is predominantly odontogenic microorganisms including involvement of streptococci and bacteroides (16). Antibiotic of choice for odontogenic infections is penicillin (17). Search of the literature has shown that other types of antibiotic that had been used in treating osteomyelitis were clindamycin, tetracycline and cephalosporins (18).

The use of gentamicin-impregnated polymethylmetacrylate (PMMA) beads in the management of long bones osteomyelitis is welldocumented (19-20). Leaching of the antibiotic from the beads by tissue fluid and blood ensures sufficiently high local levels to control and often eliminate the infection with minimal systemic accumulation (23).

There is little published evidence to determine the most effective duration of antibiotic course. Data from animal models showed that bacteria could be cultured from infected bone even after two weeks of antibiotic therapy (24). Lazzarini et al (18) reviewed the literature on osteomyelitis and observed that two major trends were found - that the majority of the patients were treated with antibiotic therapy for about six weeks while some were prescribed antibiotics for about six months. They concluded that despite three decades of research, the available literature on the treatment of osteomyelitis is inadequate to determine the best agent(s), route and duration of antibiotic therapy.

In this case, the patient was treated with parenteral cloxacillin $250 \mathrm{mg}$ qid for two weeks postoperatively. In addition, gentamicin was also applied locally to the surgical site for one week. Even though the duration appeared shorter than recommended in the literature, the infection healed well at the end of the antibiotic regime. Parenteral route of administration of antibiotic was decided as opposed to oral route as it was felt that compliance to antibiotic therapy as well as maintenance of cleanliness of surgical site was doubtful in this patient.

Most authorities believed that an incompletely debrided bone infection is prone to treatment failure, no matter what type of antibiotic has been used (25). Simpson et al (26) were also of the opinion that radical debridement of infected and necrotic bone is even more important in the compromised host. However, in this patient, the lower border of mandible was dangerously thin and excessive debridement could lead to pathological fracture, thus the treatment formulated tried to achieve a balance between these factors.

Currently, the child is under three-monthly routine dental follow-up. The options for correcting the dentofacial deformities had been discussed with his parents but at the present time, they are not keen on any elective procedures.

At this stage, it is not possible to conclude the reason for the complications that occurred after the second general anaesthetic session. Based on the swelling and the immediate response to chlorpheniramine, it is most likely due to an allergic reaction. As the patient is not allergic to latex, he may be allergic to the anaesthetic agents or antibiotics and further tests will be conducted as soon as the parents and patient are willing to undergo the procedure.

\section{CONCLUSION}

Extractions and other dental procedures in the jaws in pyknodysostosis patients can lead to serious complications such as osteomyelitis. In order to prevent these complications, extractions should be carried out with antibiotic coverage. However, if osteomyelitis develops, early combined surgical and antibiotic treatment is indicated.

\section{REFERENCES}

1. Francisco JV, Nicholoff TJ. Pyknodysostosis: An unusual presentation in a denture wearer. A case report. Oral Surg Oral Med Oral Pathol 1999; 72: 693-5.

2. Bathi RJ, Masur VN. Pyknodysostosis - a report of two cases with a brief review of the literature. Int J Oral Maxillofac Surg 2000; 29: 439-42.

3. Gelb BD, Shi G-P, Chapman HA. Pycnodysostosis: a lysosomal disease caused by cathepsin -K deficiency. Science 1996; 273: 12368. 
4. Karkabi S, Reis ND, Linn S (1993). Pyknodysostosis: Imaging and laboratory observations. Calcif Tissue Int 1993; 53: 170173.

5. Everts V, Aronson DC, Beertsen W. Phagocytosis of bone collagen by osteoclasts in two cases of pycnodysostosis. Calcif Tissue Int 1985; 37: 25-31.

6. Hunt NP, Cunningham SJ, Adnan N, Harris M. The dental, craniofacial and biochemical features of pyknodysostosis: A report of three new cases. J Oral Maxillofac Surg 1998; 56: 497 504.

7. Illankovan V, Moos KF. Pyknodysostosis: Case report with surgical correction of the facial deformity. Br J Oral Maxillofac Surg 1990; 28: 39-42.

8. Gorlin RJ, Cohen MM, Levin CS. Syndromes of the head and neck. Oxford: Oxford University Press. 1990; 232-4, 249-52, 285-7.

9. Iwu CW. Bilateral osteomyelitis of the mandible in pycnodysostosis. A case report. Int $\mathbf{J}$ Oral Maxillofac Surg 1991; 20: 71-2.

10. Schmitz JP, Gassmann CJ, Bauer AM, Smith BR. Mandibular reconstruction in a patient with pyknodysostosis. J Oral Maxillofac Surg 1996; 54: 513-7.

11. Muto T, Mishia H, Taira H, Murose H, Kanazawa M. Pyknodysostosis. Report of a case and review of Japanese literature with emphasis on oral and maxillofacial findings. Oral Surg Oral Med Oral Pathol 1991; 72: 449-55.

12. Zachariades N, Koundoris I. Maxillofacial symptoms in two patients with pycnodysostosis J Oral Maxillofac Surg 1984; 42: 819-23.

13. Grime PD, Bowerman JE, Weller PJ. Gentamicin impregnated polymethlmetacrylate (PMMA) beads in the treatment of primary chronic osteomyelitis of the mandible. Br J Oral Maxillofac Surg 1990; 28: 367-74.

14. Steiner M, Gould AR, Means WR. Osteomyelitis of the mandible associated with osteopetrosis. J Oral Maxillofac Surg 1983; 41: 395-405.

15. Van Merkesteyn JP, Bakker DJ, Van der Waal I et al. Hyperbaric oxygen treatment of chronic osteomyelitis of the jaws. Int J Oral Surg 1984; 13: 386-95.
16. Koorbusch GF, Fotos P, Goll KT. Retrospective assessment of osteomyelitis. Etiology, demographics, risk factors, and management of 35 cases. Oral Surg Oral Med Oral Pathol 1992; 74: 149-54.

17. Petersen LJ, Ellis E, Hupp JR, Tucker MR (2003). Complex odontogenic infections. In Contemporary Oral and maxillofacial Surgery 4th Ed. St. Louis: Mosby. 367-79.

18. Lazzarini L, Lipsky BA, Mader JT. Antibiotic treatment of osteomyelitis: what have we learned from 30 years of clinical trials? Int J Infect Dis 2002; 9: 127-38.

19. Hook M, Lindberg L . Treatment of chronic osteomyelitis with Gentamicin PMMA beads: A prospective study in Nepal. Tropical Doctor 1987; 17: 157-63.

20. Evans RP, Nelson CL. Gentamicin-impregnated polymethylmethacrylate beads compared with systemic antibiotic therapy in the treatment of chronic osteomyelitis. Clin Orthop Relat Res. 1993; 295: 37-42.

21. Grime PD, Bowerman JE, Weller PJ. Gentamicin impregnated polymethylmethacrylate (PMMA) beads in the treatment of primary chronic osteomyelitis of the mandible. $\mathrm{Br} \mathrm{J}$ Oral Maxillofac Surg. 1990; 28: 367-74.

22. Ludwig Von H, Haneke A, as quoted In: Grime PD, Bowerman JE, Weller PJ. Gentamicin impregnated polymethlmetacrylate (PMMA) beads in the treatment of primary chronic osteomyelitis of the mandible. Br J Oral Maxillofac Surg 1990; 28: 367-74.

23. Alpert B, Colosi T, Von-Fraunhofer JA, Seligson D. The in-vivo behaviour of gentamicin PMMA beads in the maxillofacial region. J Oral Maxillofac Surg 1989; 47: 46-9.

24. Norden CW. Lessons learned from animal models of osteomyelitis. Rev Infect Dis 1988; 10: 103-10.

25. MaderJT, Shirtliff M, Calhoun JH. Staging and staging application in osteomyelitis. Clin Infect Dis 1997; 25: 1303-9.

26. Simpson AH, Deakin M, Latham JM. Chronic osteomyelitis. The effect of the extent of surgical resection on infection-free survival. J Bone Joint Surg Br 2001; 83: 403-7. 